
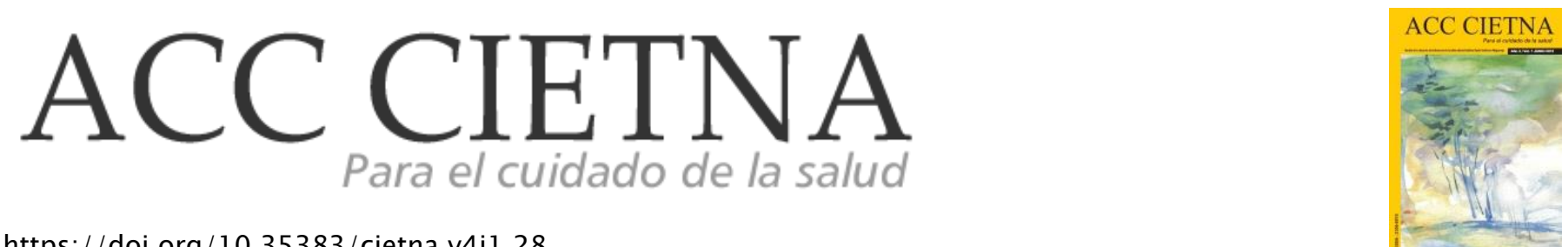

https://doi.org/10.35383/cietna.v4i1 128

\title{
Participación de la familia durante el tratamiento del paciente con tuberculosis - Tumbes
}

\author{
Julca Galindo Florinda ${ }^{1}$, Melgar Morán Carlos Christian²
}

INFORMACIÓN DEL ARTÍCULO
Historia del artículo:
Recibido el 23 de mayo de 2016
Aceptado el 15 de octubre de 2016

\section{Palabras clave:}

Participación

Familia

Tratamiento

Tuberculosis

\section{RESUMEN}

Esta investigación tuvo como objetivo: Describir y analizar la participación de la familia durante el tratamiento del paciente con tuberculosis. Su importancia radicó en que la familia es la institución donde se lleva a cabo el proceso de salud/enfermedad/atención y la tuberculosis en el Perú es considerada como un gran problema de salud pública. El marco teórico conceptual está sustentado por Trilla y Novella para participación, Bustamante para familia, tuberculosis por Farga. Desarrollada con enfoque cualitativo, como abordaje estudio de caso. Los sujetos investigados fueron 05 familiares de pacientes que padecían de tuberculosis en el Distrito de la Cruz- Tumbes, determinados por saturación. La recolección de los datos fue, mediante la guía de entrevista semiestructurada a profundidad; la información se sometió al análisis de contenido. Se ejercieron los principios éticos del Informe de Belmont y los de rigor científico. Obteniendo como categorías I. Participación consultiva de la familia durante el tratamiento antituberculoso: aconsejando a no abandonar el tratamiento y preocupándose por los exámenes de control. II. Participación proyectiva de la familia durante el tratamiento antituberculoso: Acompañando al establecimiento de salud para recibir tratamiento, preparándole una alimentación balanceada y realizando medidas de prevención. Llegando a la consideración final, que la participación de la familia durante la administración del tratamiento antituberculoso es un soporte vital y emocional para el afrontamiento de la enfermedad, pues contribuye

\footnotetext{
${ }^{1}$ Licenciada en enfermería. Enfermera asistencial. Hospital ESSalud Carlos Cortéz Jimenez - Tumbes, Tumbes, Perú. Email: xtianmelgar@gmail.com

${ }^{2}$ Docente a tiempo parcial en la Universidad Peruana Cayetano Heredia. Enfermero especialista en Cuidados Nefrológicos. Enfermero Asistencial Clinica Salud Renal SAC. Lima, Perú. Email: carlos.melgar.m@upch.pe
} 
positivamente en el paciente, evitando el surgimiento de sentimientos de culpa, rechazo o de abandono.

\section{Family participation in the treatment of patients with tuberculosis-} Tumbes

\section{ABSTRACT}

Keywords:

Participation

Family

Treatment

Tuberculosis

The objective of this research was to: Describe and analyze the participation of the family during the treatment of the patient with tuberculosis. Its importance lies in the fact that the family is the institution where the health / disease / care process is carried out and tuberculosis in Peru is considered a great public health problem. The theoretical framework is supported by Trilla and Novella for participation, Bustamante for family, tuberculosis by Farga. Developed with a qualitative approach, as a case study approach. The subjects investigated were 05 relatives of patients suffering from tuberculosis in the District of La Cruz-Tumbes, determined by saturation. The data collection was, through the semi-structured interview guide in depth; the information was submitted to content analysis. The ethical principles of the Belmont Report and those of scientific rigor were exercised. Obtaining as categories I. Consultative participation of the family during the antituberculous treatment: advising not to abandon the treatment and worrying about the control examinations. II. Projective participation of the family during antituberculosis treatment: Accompanying the health establishment to receive treatment, preparing a balanced diet and taking preventive measures. Arriving at the final consideration that the participation of the family during the administration of antituberculous treatment is a vital and emotional support for the coping with the disease, because it contributes positively to the patient, avoiding the emergence of feelings of guilt, rejection or abandonment.

\section{Introducción}

En el Perú, la tuberculosis (TB) es una importante causa de morbilidad en el grupo de jóvenes y adultos, se reportan casos en todos los departamentos del país, pero la enfermedad se concentra principalmente en los departamentos de la costa central y la selva. En los años 2013 al 2014, cinco departamentos (Madre de Dios, Ucayali, Loreto, Lima, e Ica) presentaron incidencia de TB por encima del nivel nacional, estos departamentos reportaron el $72 \%$ de los casos nuevos notificados en el país. Lima es el departamento que más casos de tuberculosis concentra en el país (60\%) y, es el tercer departamento con la incidencia más alta. La forma clínica que afecta principalmente es la pulmonar en el $85 \%$ de los casos, y el resto son tipo extrapulmonarl. A pesar de que el control de la tuberculosis se ha basado principalmente en la aplicación del tratamiento directamente observado de curso corto (DOTS - Directly Observed Therapy-Short Course). El DOTS (que enfatiza particularmente la detección temprana de los casos, la adherencia y el éxito del tratamiento), es 
considerado una de las intervenciones de salud pública más rentables jamás implementada, que ha contribuido con la reducción significativamente de la morbi-mortalidad por $\mathrm{TB}^{2}$. El tratamiento farmacológico de la tuberculosis sensible a las drogas considera dos fases, ambas supervisadas: la primera fase de inducción o bactericida (administración diaria), la segunda fase es de mantenimiento o esterilizante (administración intermitente). Esta enfermedad no discrimina y quien la llega a poseer debe ser considerado como valiente, ya que sobre su "base biológica y la de su largo y penoso tratamiento", se va a construir la enfermedad con elementos sociales y morales, que van a incidir en el proceso de tratamiento, además sobre estos pacientes también va recaer el estigma social de cómo se le debe ver "objeto indeseable" quien como fuente de contagio debe ser "rehuido"3. Este prejuicio afecta a una de las necesidades fundamentales de los humanos, la aceptación social, necesidad que para intentar satisfacer los diferentes comportamientos que dependerán de las características de la personalidad de cada individuo y del contexto en que se desenvuelve, incluida en este último la familia. Se entiende por familia al organismo vivo, complejo, cuya trayectoria de vida es un transcurrir de diversidades, adversidades, semejanzas, diferencias, individualidades, singularidades $y$ complementariedades, que lucha por su preservación y desarrollo en un tiempo-espacio y territorio dado, y al cual se siente perteneciente, interconectada y enraizada biológica, solidaria, amorosa, cultural, política y socialmente. Ver a la familia como un organismo vivo significa verla articulada en su ambiente natural y con los otros organismos sociales a fin de mantenerse como familia. $Y$ como sujeto de la investigación, la familia viene a ser una entidad compuesta por seres humanos que se encuentran unidos por lazos de sangre, afecto, una misma cultura y un sinnúmero de valores y virtudes característicos de cada grupo humano, que en su labor cotidiano, viven experiencias positivas y negativas que engrandecen sus lazos y que juntos actúan para salir adelante en relación al tiempo y al lugar donde se encuentren ${ }^{4}$. En toda familia existe la dependencia de sus miembros entre sí, tanto que cuando uno de ellos se enferma todos los demás miembros experimentan alarma, puesto que la enfermedad amenaza la integridad familiar, sumándose el temor al contagio, sentimientos de vergüenza e inseguridad, determinando actitudes hostiles y tipos de conducta que amenazan los mecanismos físicos y psicológicos de sostén que actúan dentro de la familia ${ }^{5}$. La problemática social de esta enfermedad actualmente se centra en el riesgo de incumplimiento terapéutico y su posible influencia negativa tanto en la salud del paciente como en el control de la enfermedad desde el punto de vista de la salud pública ${ }^{6}$. Si bien el profesional de la salud juega un papel importante en el cumplimiento del tratamiento por parte del paciente, la participación de la familia es la clave para hacer frente a la enfermedad, porque influye en la adopción de hábitos, estilos y comportamientos que son relevantes para que el proceso terapéutico tenga éxito7. "Participación" (del latín participatio, -ônis) es, según el Diccionario de la Real Academia Española, "acción y efecto de participar;" y para este verbo (participâre) ofrece diversas acepciones: "tomar parte en algo"; "compartir, tener las mismas opiniones, ideas, etc., que otra persona"; y "dar parte, noticiar, comunicar." Por ello, Participar puede significar hacer acto de presencia, tomar decisiones, estar informado de algo, opinar, gestionar o ejecutar; desde estar simplemente apuntado a, o ser miembro de, a implicarse en algo en cuerpo y alma8.

En la práctica, la participación familiar puede tener un trasfondo de confianza o de desconfianza, de 
colaboración o de enfrentamiento, de personalización o de masificación. Cuando domina lo negativo, la participación se convierte en un medio desagradable y educativamente ineficaz. En este sentido se puede decir que la participación es la forma de realizar la solidaridad. Para lograr la solidaridad es conveniente poner en práctica una supervisión positiva y entusiasta que anime a realizar las tareas encomendadas, entrando a tallar tipos de participación como son: la consultiva y la proyectiva.

Siendo responsabilidad del personal profesional de enfermería la Estrategia Sanitaria de Control y Prevención de la Tuberculosis, estos deben brindar una atención Integral e Individualizada dirigida al paciente, familia y comunidad; poniendo énfasis en la "comunicación", porque favorecerá el comportamiento y estilos de vida saludables", abordando también la educación, control y seguimiento del enfermo con tuberculosis $y$ contactos; pero, no solo ver el aspecto biológico, sino también el aspecto emocional del paciente; puesto que sobre la tuberculosis aun recae el "estigma social que va a ser un gran peso emocional para quien la sufre y su familia" y sobre todo, observar la participación que muestra la familia y la influencia que tienen en el paciente, este soporte emocional tan importante para el afrontamiento exitoso o no de la enfermedad ${ }^{9}$.

A esta realidad, se suma el hecho de haber observado la poca participación de los familiares durante el proceso y tratamiento de la tuberculosis de sus pacientes, Surgiendo con ello la siguiente interrogante: ¿Cuál es la participación de la familia durante el tratamiento del paciente con tuberculosis? El objeto de investigación fue, la participación que tiene la familia durante el tratamiento antituberculoso desde la percepción de los familiares y la persona que padece de tuberculosis. Planteándose como objetivos: describir y analizar la participación de la familia durante el tratamiento del paciente con tuberculosis. La presenta investigación se justifica, porque la familia establece el contexto más inmediato en el que se desenvuelve la enfermedad, es el soporte social que va a actuar de manera amortiguadora entre eventos estresantes de la vida y la enfermedad. Favorece el pronóstico de mejoría del individuo; pero, en otras situaciones puede ocurrir una mutua culpabilidad. La enfermedad de alguno de los integrantes de la familia produce un desequilibrio de todo el grupo, esto va a depender de la naturaleza de la enfermedad, de la manera como se toma o encara y de las consecuencias sociales y psicológicas y es allí cuando se pueden dar situaciones, como que se incremente $y$ fortalezca los lazos familiares.

\section{Metodología}

Fue un estudio de tipo cualitativo, porque se enfoca a comprender y profundizar los fenómenos, explorándolos desde la perspectiva de los participantes en un ambiente natural y en relación con el contexto, utilizando la recolección de datos sin medición numérica para descubrir o afinar preguntas de investigación en el proceso de interpretación ${ }^{10}$. El abordaje metodológico fue estudio de caso'l, es una investigación a profundidad de una sola entidad o de una serie reducida de entidades. Normalmente la entidad es un individuo, pero también puede tratarse de familias, grupos, instituciones $u$ otras entidades sociales individuales. Analiza $y$ comprende variables para la historia, desarrollo o asistencia para los problemas del o de los sujetos, que es lo que piensa, como se desarrolla, donde se centra en entender el fenómeno específico, contexto, como la participación de la familia durante el tratamiento del paciente con tuberculosis. En el desarrollo de este trabajo, se tomaron en cuenta los siete principios del 
estudio de caso propuestos, por Menga y Ludke ${ }^{11}$. Además se cumplió con las tres fases para la credibilidad a la investigación. La primera fase es la exploratoria, el estudio de caso se inició con un plan, surgió al plantearse la interrogante ¿Cómo es la participación de la familia durante el tratamiento del paciente con tuberculosis?, que emergió durante la atención otorgada a los pacientes con diagnóstico de tuberculosis que acudían al programa a recibir tratamiento al establecimiento; actividad inmersa durante el servicio rural urbano marginal de la investigadora. Posteriormente se procedió a revisar la literatura relacionada con el tema, que sirvió de base para replantear las interrogantes y esclarecer algunos puntos con la finalidad de descubrir el objetivo de la investigación, dándose a medida que avanzaba el estudio. Durante la segunda fase, delimitación del estudio, se procedió a recolectar la información necesaria de modo sistémico, utilizándose la entrevista como técnica para registrar los datos de forma completa. Antes de proceder a recolectar los datos, se tuvo en claro los límites del estudio, es decir únicamente se investigó la participación de la familia durante el tratamiento del paciente con tuberculosis. Las respuestas fueron registradas en una grabadora de voz, que garantizó la fidelidad de la información recolectada y una visión profunda del fenómeno.

Finalmente, en la tercera fase, análisis y elaboración del informe, se realizó el análisis de contenido a los datos encontrados, permitió una mayor compresión del discurso, al transcribirse las entrevistas, se extrajeron las unidades de significado, que permitieron establecer las categorías. Por último, se elaboró el informe de modo sucinto, utilizando un lenguaje sencillo de fácil entendimiento para el lector. Los sujetos investigados, fueron los familiares de los pacientes asegurados con tuberculosis en el Distrito de la Cruz, Tumbes. El escenario de estudio, fueron sus viviendas. El total de personas que participaron en el estudio, fueron 7 determinadas por los criterios de saturación y redundancia, a quienes se les asignó una letra más un número como seudónimo para proteger su identidad.

La recolección de los datos, fue mediante la guía de entrevista semiestructurada individual a fondo. La información se sometió a un proceso de análisis de contenido temático que comprendió tres etapas. Durante el desarrollo de la investigación científica se tuvo en cuenta los principios éticos de beneficencia, respeto a la dignidad humanaautodeterminación, justicia y privacidad que postula el informe Belmont ${ }^{12}$. Así como los criterios el rigor científico: Credibilidad o valor de verdad, aplicabilidad o transferencia, consistencia o dependencia y neutralidad o confirmación ${ }^{10}$.

\section{Resultados, análisis y discusión}

Después de la descontextualización de los discursos y su respectivo análisis, emergieron las siguientes categorías.

Primera: Participación consultiva de la familia durante el tratamiento antituberculoso, con sus respectivas subcategorías: Aconsejando a no abandonar el tratamiento y preocupándose por los exámenes de control.

Segunda: Participación proyectiva de la familia durante el tratamiento antituberculoso, con sus respectivas subcategorías: Acompañando al establecimiento de salud para recibir tratamiento; preparándole una alimentación balanceada y realizando medidas de prevención.

Participación consultiva de la familia en el tratamiento antituberculoso.

La participación consultiva, supone escuchar la palabra de los sujetos. No son meros espectadores, ejecutantes o usuarios de algo previa y externamente decidido; sino, que se les demanda su parecer sobre asuntos que de forma directa o indirecta les conciernen. Se les alienta a 
opinar, proponer o valorar y se facilitan canales para ello. Cuando un sujeto o un colectivo hacen oír su voz sobre un asunto determinado sin que quien ostenta el poder se lo haya solicitado, está expresando su opinión sobre tal asunto y además está metaexpresando que se le escuche ${ }^{8}$. Dentro de este contexto emergió la siguiente subcategoría:

Aconsejando a no abandonar el tratamiento.

Se define como abandono, a la inasistencia continua al tratamiento farmacológico durante un mes o más. El abandono de tratamiento de la tuberculosis, es uno de los factores más importantes, que disminuye la eficiencia de la terapia, asociándose a fracasos, mayor mortalidad, desarrollo de resistencia bacteriana y un período de contagiosidad más prolongado13, 14. La familia es la primera que entra en contacto con el individuo y el que más influencia ejerce sobre él, es la mayor fuente de apoyo social y personal de que pueden disponer las personas ${ }^{15}$ como lo expresan los siguientes discursos:

“...Todos los días le repetía a mi hijo que continúe con el tratamiento, que sea paciente... Que no tenga prisa..." - E3.

“...No había día que le dijera a mi esposo que no deje el tratamiento, más por sus hijos..." - El.

“...a mi hermano siempre le daba fuerzas para que no abandone el tratamiento y siempre me hizo caso..." - E2.

La tuberculosis pulmonar es curable; pero, es indispensable no abandonar el tratamiento, al suspenderlo, la enfermedad se empeora rápidamente y causa que el bacilo se haga resistente a los medicamentos ${ }^{16}$. Los discursos reflejan, la participación consultiva mediante la actitud que toma la familia aconsejando al paciente a no abandonar el tratamiento, situación que contribuye positivamente en la curación de la enfermedad, permitiendo un adecuado afrontamiento de la enfermedad, teniendo en cuenta que existirán cambios en el estilo de vida. Para evidenciar la eficacia del tratamiento farmacológico se realizan exámenes de control surgiendo así la siguiente subcategoría. Preocupándose por los exámenes de control.

Los exámenes de rutina durante el tratamiento de la tuberculosis (laboratorio, radiografías, etc), tienen una capital importancia; no se exagera, cuando se afirma que el laboratorio bacteriológico es la piedra angular de toda la lucha antituberculosa ${ }^{17}$

“...todos los meses estaba pendiente de sus pruebas de esputo, de su peso, su radiografías..." - El.

“...también iba con él cuando le tocaban sus exámenes de control..." - E4.

"...estaba pendiente de sus controles para saber el estado de su salud..." - E6.

Preocuparse por los exámenes de control, es una actitud de la familia que va a contribuir positivamente en esta nueva situación, ya que cada nuevo examen que se realice, va a mostrar un nuevo panorama en cuanto a recuperación y por consiguiente un nuevo y adecuado afrontamiento de la enfermedad, que contribuirá a una rápida recuperación.

Participación proyectiva de la familia en el tratamiento antituberculoso.

En la participación «proyectiva», el sujeto no se limita a ser un simple usuario, sino que hace algo más que opinar desde fuera: se convierte en agente. Esta condición de agente que se le reconoce, es quizá lo que mejor caracteriza tal forma de participar. Lo esencial, en esta clase de participación, es que los sujetos consideren como propio el proyecto y que puedan intervenir en él, desde dentro8. Planteamiento que permite el surgimiento las siguientes sub categorías:

Acompañando al establecimiento de salud para recibir tratamiento.

El núcleo familiar, es el ambiente más cercano en donde el paciente con tuberculosis, recibe apoyo; si en este núcleo hay desintegración y se margina a la persona enferma, se le hace sentir inútil y culpable. El paciente enfermo por TB se sentirá 
rechazado y no tendrá motivación para continuar el tratamiento ${ }^{18}$. Por ello, cuando un familiar acude junto con el paciente al establecimiento de salud para recibir tratamiento, recrea un ambiente de ayuda, confianza y motivación, para continuar este largo periodo, siendo reflejado en los siguientes discursos:

“...siempre iba con mi hijo al seguro para que reciba su tratamiento..." - E3.

“...lo acompañaba al seguro para que tome sus pastillas..." - E4.

“...los primeros meses del tratamiento iba acompañando a mi hermano..." - E2. "...cuando mi mujer iba a tomar las pastillas yo siempre la acompañaba..." - E5.

Manjarréz y otros ${ }^{19}$, comprobaron que dentro de las principales causas de abandono del tratamiento antituberculoso se encuentra la deficiente identificación entre el grupo familiar y el equipo médico, lo que deja en evidencia el papel fundamental que juega la familia en la adherencia al tratamiento. Cuando el paciente percibe el apoyo, ayuda y preocupación de la familia, se sentirá más confiado y motivado a culminar con el tratamiento para poder recuperarse pronto $y$ reinsertarse a su vida cotidiana ${ }^{20}$, es el soporte social más importante, contribuye a fortalecer su autoestima, valoración y compromiso para entrar en el largo tratamiento antituberculoso. De lo contrario, el abandonado por parte de la familia, genera en ellos frustración, retracción social y depresión ${ }^{21}$. Por ello, la unión familiar es beneficiosa, ya que se evita que el paciente no tenga sentimientos de culpa, rechazo o de abandono respecto al tratamiento, tal como lo han manifestado en las entrevistas.

\section{Preparándole una alimentación balanceada}

Para que el tratamiento de tuberculosis sea exitoso, debe estar acompañado de una dieta balanceada y completa, rica en proteínas, consumir bastantes frutas y verduras, sobre todo las de color amarillo que contribuyen a mejorar su sistema inmunológico; en otras palabras, la dieta debe ser saludable:

“...estaba pendiente de su alimentación... le hacía menestras todos los días para que coma..." - El.

"...Le hacía hígado de pollo, concentrado de bazo, le daba frutas, menestras, para que coma..." - E3.

"...le daba de comer menestras lentejitas negras y verdes, carne, hígado, jugos de betarraga, zanahoria..." - E6.

Es importante, que la familia del paciente con tuberculosis, brinde una dieta balanceada, rica en proteínas y vitaminas, a fin de contribuir a la regeneración tisular de los pulmones, producto de la infección bacteriológica; la misma que debe contener: menestras en sus diversas variedades, productos animales y sus derivados, frutas $y$ verduras, y además brindarle el apoyo emocional constante, para poder cumplir con el tratamiento ${ }^{22}$. La familia puede ejercer un efecto sobre la salud de sus miembros; desde el punto de vista conductual, hace referencia a las prácticas de salud que ejercen influencia sobre la persona. Así, algunos comportamientos relacionados con la salud pueden ser la dieta, el ejercicio, el descanso y sueño ${ }^{23}$.

\section{Realizando medidas de prevención}

El Ministerio de Salud hace hincapié en el trabajo conjunto, a fin de desarrollar acciones destinadas a generar una demanda informada y satisfecha en la prevención de la tuberculosis, motivando a la población a tomar comportamientos preventivos, para asegurar el bienestar de la familia y la comunidad, a través de una eficaz intervención comunicacional.

"...yo le compré a mi hijo pañuelos descartables para cuando tosa, se cubra y los bote..." - E2.

"...cada vez que mi marido tosía o estornudaba, le decía que se cubra para evitar contagiar a los niños..." - E4.

"...cada vez que tosía, le decía que se lave las manos, y que se cubra..." - E5.

A pesar que en la actualidad aún hay un predominio del nivel medio - bajo de 
conocimientos generales sobre la tuberculosis, estos son insuficientes para prevenir y enfrentar la enfermedad acorde a nuestro desarrollo, si analizamos que casi la mitad de la población de las zonas rurales carece de conocimientos e información. Se considera que al estar la población informada sobre tuberculosis, mediante la difusión y conocimiento de la enfermedad, coadyuva a que la población se apropie del problema y contribuya a disminuir los riesgos y daños a la salud; poniendo en práctica todos la información sobre prevención de la enfermedad, en la medida que la reciben; esto se evidencia en los discursos de los participantes. ${ }^{19}$ El soporte familiar y social es un eje importante en la recuperación del paciente, ya que el paciente se siente más confiado y motivado a culminar con su tratamiento para recuperarse pronto y reinsertarse a su vida cotidiana ${ }^{24}$.

\section{Conclusiones}

La familia durante el periodo del tratamiento antituberculoso es un soporte vital y emocional para el afrontamiento de la enfermedad, ejerciendo la participación consultiva a través de aconsejar al familiar, a no abandonar el tratamiento y preocuparse por los exámenes de control; así pues, ser su apoyo, realizar muestras de solidaridad, cariño, afecto, contribuye positivamente en el paciente, evitando el surgimiento de sentimientos de culpa, rechazo o de abandono del tratamiento.

La familia tiene una participación proyectiva, en el tratamiento antituberculoso, que permite el involucramiento con el familiar enfermo, acompañándolo para acudir al establecimiento de salud, de manera permanente. Además, de contribuir al mantenimiento del estado nutricional de los pacientes con tuberculosis, brindando una alimentación balanceada, tomando las medidas preventivas del caso, para evitar la proliferación del bacilo de Koch.
Por ello, se recomienda a las autoridades de los establecimientos de salud públicos o privados, basándose en los resultados de la presente investigación, elaboren estrategias, talleres, charlas educativas, que permitan mejorar la relación familia-paciente, siendo un ente fundamental durante el transcurso de la enfermedad.

A los profesionales de enfermería promuevan la participación activa de la familia, bajo el asesoramiento, la supervisión, el seguimiento durante el transcurso del tratamiento de la enfermedad.

\section{Bibliografía}

1. Ministerio de Salud del Perú. Análisis de la situación epidemiológica de la tuberculosis en el Perú. 2015 Disponible en: http://www.dge.gob.pe/portal/docs/tools /t bc/asistbc.pdf

2. Hargreaves JR, Boccia D, Evans CA, Adato $M$, Petticrew M, Porter JDH. The social determinants of tuberculosis: from evidence to action. Am J Public Health. abril de $2011 ; 101$ (4):654-62.

3. Ministerio de salud - República del Peú; Dirección General de la salud de las personas; Lima 2006; Construyendo

Alianzas Estratégicas para detener la Tuberculosis - La Experiencia Peruana.

Disponible en

URL:http://bvs.minsa.gob.pe/local/dgs p/145_construy2.pdf

4. Bustamante S. Principios de cuidado a partir del saber in común de las Familias. Facultad de Enfermería - Univ. Nac. De Trujillo, 2004.

5. Balcázar-Rincón LE, Ramírez-Alcántara $Y \mathrm{~L}$ , R o d ríg u e z M. D e p r e s i ó n y funcionalidad familiar en pacientes con diagnóstico de tuberculosis. Rev Esp Med Quir 2015; 20:135-143. 
6. Heredia M, Puc M, Caamal A, Vargas A. Determinantes sociales relacionados con el tratamiento de tuberculosis. Revista Biomed. 2012. (Citado el 04 de febrero del 2014) ; 23 (3) .Disponible en : http://www.medigraphic.com/pdfs/revbio /bio-2012/biol23e.pdf

7. De Almeida J, Soares D, Duarte L, Alves C, Santana, L, Scatena $T$. et al. Enfoque familiar e orientação para a comunidade no controle da tuberculose. Rev Bras Epidemiol $2011 ; 14(2): 207-16$.

8. Trilla J, \& Novella A. Educación y participación social de la infancia. Revista Iberoamericana de Educación. 2001. 26, 1 $37-164$. R e t ri e v e d f rom http://www.rieoei.org/rie26f.html

9. Zárate E. Tuberculosis en Nuevos Escenarios: Establecimientos Penitenciarios. P.2, 9. Perú. 2005.

10. Hernández R, Fernández C, Baptista P. Metodología de la investigación. Quinta edición. McGRaw-Hill Interamericana Editores: México. 2010.

11. Lüdcke M. El Estudio de Caso su Potencial en Educación. Brasil. Traducción por Lucia Aranda Moreno. 1986. Disponible en URL: http:/ / www.enfermeria.sld.cu/socuenf/ congresoresumen 07 /docencia.html

12. Informe Belmont. Principios Éticos y Directrices Para La Protección De Sujetos Humanos de Investigación. Reporte de la Comisión Nacional para la Protección de Sujetos Humanos de Investigación Biomédica y de Comportamiento. 1976. Disponible en URL: http://www.censida.salud.gob.mx/des cargas/etica/Informe_Belmont-112008.pdf

13. Vijay S, Kumar P, Chauhan L S, Vollepore B $\mathrm{H}$, Kizhakkethil U P, Raos G. Risk factors associated with default among new smear positive TB patients treated under DOTS in India. Pai M, editor. PLoS One [Internet]. Public Library of Science; 2010, 5 (4):de
10043 .Disponible

en:

http://dx.plos.org/10.1371/journal. pone. 0010043 .

14. Farga V. Tuberculosis, lo que hay que saber. Rev Chil Enferm Respir 2008, 26; 24: 317- 22 .Disponible en: http://www.scielo.cl/pdf/rcher/v24n4/art 0 8.pdf

15. Poveda R. Cuidados a Pacientes CrónicosAspectos Psicosociales de la Enfermedad Crónica. 2010. Disponible en URL: http://www.aniortenic.net/apunt_cuidad_c ronic_2.htm

16. Organización Mundial de la Salud. Tuberculosis. Ginebra: Organización Mundial de la Salud; 2014 (Citado el 10 de Enero del 2014). Disponible en: http://www.who.int/mediacentre/factshee ts/fs104/es/

17. Norma técnica de salud para el control de la tuberculosis. 2006. Dirección general de salud de las personas. Estrategia sanitaria nacional de prevención y control de la tuberculosis. Lima, Perú. Pág. 62 - 70.

18. Salas P. Horcado Chamizo García. Determinantes sociales de la adherencia al tratamiento de la Tuberculosis: Una Discusión desde la perspectiva heurística del riesgo. Gestión Vol.10 N ${ }^{\circ} 1$ Primer semestre 2002.

19. Manjarréz E. Principales causas de abandono del tratamiento contra la tuberculosis pulmonar. Gaceta Méd Méx.Vol 129. No 1.EnFeb 1993. P 60.

20. Fabelo J, Iglesias S, Bachir S, Ramírez A, Amador J. Percepción de apoyo social por pacientes con enfermedad alcohólica en fase de recuperación. Rev. Hosp. Psiquiátrico de la Habana. 2012; 9(1). Disponible en:

http://www.revistahph.sld.cu/rev12012/hph07112.html

21. Torres M. Estudio comparativo en enfermos con tuberculosis pulmonar frotis positivo de los grupos nunca tratados, antes 
tratados y crónicos en sus niveles de depresión y soporte social". En Tesis para obtener el título de Lic. En Psicología .U. N. M. S. M. 2002. Lima - Perú.

22. Ministerio de Salud. Instrucciones para el Personal de Dirección del Programa Nacional de Control de la Tuberculosis. P.3-5. Lima, Perú. 2004.

23. Campbell T.L \& Treat DE. The family's influence on health. In R.E. Rakel (Ed.) Texbook of family practice (). Philadelphia: Saunders Company. 1990.

24. Quevedo L, Sánchez R, Villalba F, Velásquez D. Relación del soporte familiar y social en el cumplimiento del tratamiento de pacientes con tuberculosis pulmonar en centros de salud. Rev enferm Herediana. 2015; 8(1):11-16. 